\title{
GA-Based PI-PD Controller for TCP Routers
}

\author{
R. K. Sundaram and P. K. Padhy
}

\begin{abstract}
Active Queue Management (AQM) is a key congestion control mechanism for reducing the packet loss and improving the network utilization in TCP/IP networks. This paper discusses previously developed PI controller for AQM and proposed a new GA-based PI-PD controller. It is able to control present congestion reactively and also avoid the incipient congestion proactively. Fast response to the changing traffic is achieved by PD to maintain a desired queue length, high link utilization and low packet loss. The optimized tuning parameters are determined by selection of an Integral Square Time Error (ISTE) as an objective/fitness function. We use $n s$ simulation to demonstrate the practical advantage of using GA-based PI-PD controller over PI in managing constant queue length.
\end{abstract}

Index Terms-Congestion control, genetic algorithm (GA), integral square time error (ISTE), transmission control protocol (TCP).

\section{INTRODUCTION}

Congestion control and congestion avoidance is very active research area in computer networking. After the development of TCP/IP, the network has suffered from congestion collapse and the need of most adequate AQM controller is felt. So, AQM scheme is implemented in router to signal the congestion by discarding/marking packets. Objectives of any AQM are: to stabilize the buffer queue length, maintain the constant queuing delay and to maximize the link utilization. Especially, well established random early detection (RED) [1] is a queue based AQM scheme which introduces a control mechanism by randomized packet dropping and a queue length averaging technique. To realize the above objectives RED must be able to stabilize the queue length at a desired target so as to present a predictable queuing delay and to avoid the occurrence of queue overflowing and under-flowing.

After the development of a fluid-flow model of the TCP and RED [2] using stochastic theory, control engineers have shown much interest to control the internet traffic. This model represents the behavior of the characteristic variables of the network and shows that it accurately captured the qualitative evolution of TCP traffic flows. Based on this model, a lot of research [3]-[5] and [6] has been done to find the proper tuning parameters of RED. The key requirement in selecting the appropriate parameters is the stability of TCP/RED systems. A proportional-integral controller [3] based on linearized fluid-based TCP/AQM model was

Manuscript received November 28, 2012; revised May 18, 2013. This work was supported in part by the Department of Science and Technology (DST), New Delhi India (Ref-SR/FTP/ETA-39/2007).

R. K. Sundaram is with Indian Institute of Technology, Kanpur, India (e-mail: sravi@iitk.ac.in).

P. K. Padhy is with PDPM-IIITDM, Jabalpur (e-mail: prabin16@iiitdmj.ac.in). developed to regulate the queue level, round-trip time (RTT), and packet loss. However, PI controller is sluggish with taking too much time to achieve the target queue length due to PI controller. It improves the steady state-error at the cost of an increase in rise time [3]. Therefore, in order to overcome the drawbacks of PI controller, several new modified methods are proposed. A Smith Predictor based PI (SPPA) controller [4] was presented to reduce the effect of time delays in the control loop by a predictor.

The Genetic Algorithm (GA) can be viewed as general-purpose optimization method and have been successfully applied to search, optimization and machine learning task [7]. In this paper real coded GA is adopted to find the proper tuning parameters of different controller parameters and computation is done with the use of GA's command in Matlab.

We are able to employ the feedback of current queue length through PD controller to affect the packet marking probability and control the incipient congestion in the network. In the structure, PD controller is used in the inner feedback loop to make the controller more active and faster in controlling queue length oscillation. Also, it helps to reduce the noise and to avoid the derivative kick

The paper is organized as follows. Section II discusses the linearized control system of TCP. A PI-PD controller for AQM is suggested and illustrated in Section III. Section IV shows the result of simulation to demonstrate the performance of the proposed controller scheme and also compares its performance with the PI controller. Finally brief conclusions are provided in Section V.

\section{SYSTEM DESCRIPTION}

A window-based linearized fluid-flow dynamic model [2] is considered in this study. This model reflect the dynamics of TCP accurately with the average TCP window size and the average queue length by following coupled non-linear differential equations :

$$
\begin{aligned}
& \dot{W}(t)=\frac{1}{R(t)}-\frac{W(t)}{2} \frac{W(t-R(t)}{R(t-R(t))} p(t-R(t)) \\
& \dot{q}=\left\{\begin{array}{l}
-C+\frac{N(t)}{R(t)} W(t), q>0 \\
\max \left\{0,-C+\frac{N(t)}{R(t)} W(t)\right\}, q=0
\end{array}\right.
\end{aligned}
$$

where $W$ is average TCP window size (packets), $q$ is average queue length (packets), $R(t)$ is round-trip time equalto $\frac{q(t)}{C}+T_{p}$ (secs), $C$ is the link capacity (packets/sec), $T_{p}$ is propagation delay (secs), $N$ is the 
number of TCP connections, and $p$ is the probability of packet mark/drop. For further discussion, $R(t)$ is taken as $R_{0}$. Fig. 1 shows the block diagram as represented by differential equation (1).

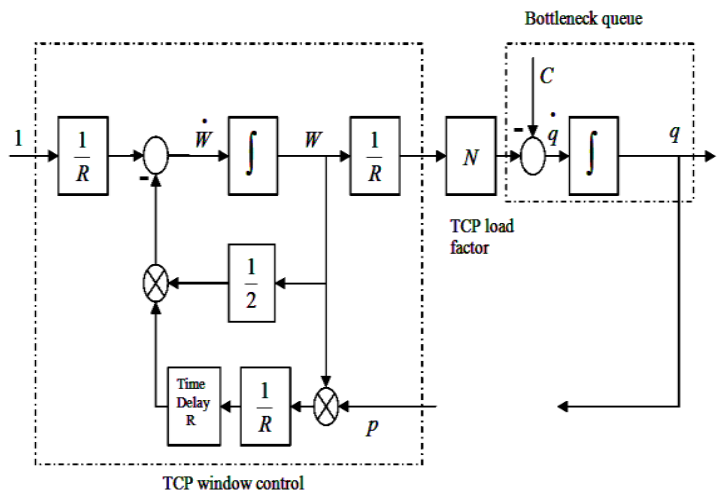

Fig. 1. Block-diagram of TCPs congestion-avoidance mode.

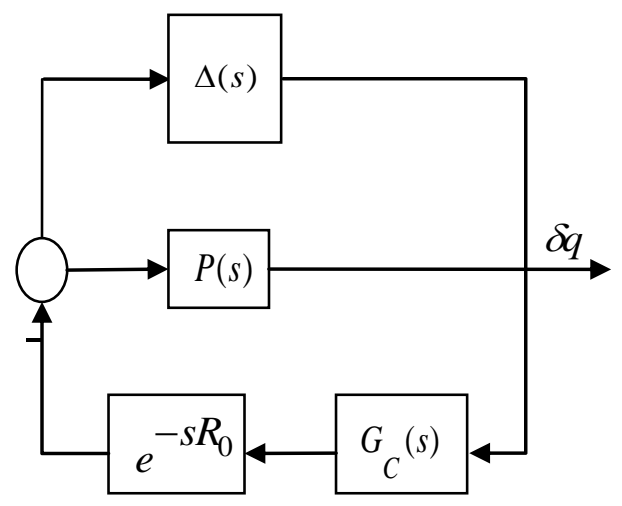

Fig. 2. AQM as a feedback control.

To carry out system analysis, we follow the linearized model [3] around the equilibrium point $\left(W_{0}, q_{0}, p_{0}\right)$ which is depicted in Fig. 2. In this model $P(s)$ is the process dynamic of TCP/AQM system which is required to control and $G_{C}(s)$ is the controller. $R_{0}$, the round trip time causes the delay in the feedback of losses. $P(s)$ and residual $\Delta(s)$ are given by

$$
P(s)=\frac{K}{\left(T_{1} s+1\right)\left(T_{2} s+1\right)}
$$

where, $K=\frac{R_{0}{ }^{3} C^{3}}{4 N^{2}}, T_{1}=\frac{R_{0}{ }^{2} C}{2 N}, T_{2}=R_{0}$ and

$$
\Delta(s)=\frac{2 N^{2} s}{R_{0}^{2} C^{3}}\left(1-e^{-s R_{0}}\right)
$$

The residual $|\Delta(j \omega)|$ shows its influence at high frequency.

\section{AQM USING PI-PD CONTROLLER}

A PI and PD controller has a transfer function of the form
$G_{C 1}(s)=K_{P} \frac{\frac{s}{z}+1}{s} \quad$ and $\quad G_{C 2}(S)=K_{f}\left(1+T_{D} s\right)$ respectively. The PI-PD controller along with the non-linear TCP/AQM dynamics is shown in Fig. 3 where $q_{0}$ is the target queue length. The consequence of derivative term is that the probability of mark/drop gets directly affected by rate of disturbance in current queue length.

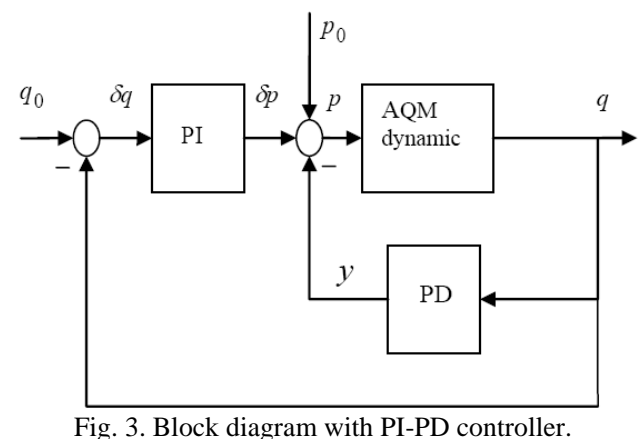

Here, a GA based PI-PD controller is proposed to achieve the desired queue length efficiently and provide the robust performance with dynamic input. A PI-PD controller generates the term $p(t)$ as a control input in (1) to guarantee the stability of the system (2). Moreover, the output error signal is defined as $e(t)=q(t)-q_{o}$, where $q_{o}$ denotes the reference queue length. A PI-PD controller with inputs $e(t)$ and $q(t)$, and the output $p(t)$ are expressed as follows: $p(t)=K_{P}\left[e(t)+\frac{1}{T_{I}} \int_{0}^{t} e(\tau) d \tau\right]-K_{f}\left[q(t)+T_{D} \frac{d q(t)}{d t}\right]$

where $K_{P}$ and $K_{f}$ are the proportional gain, $T_{I}$ and $T_{D}$ are the integral and derivative time constants, respectively.

In order to measure the performance of the closed-loop control system, an Integral Square Time Error is employed as the objective function $(O F)$.

$$
O F=I S T E=\int_{0}^{\infty} t e(t)^{2} d t
$$

The objective function will depend on controller parameters. $K_{P}, K_{f}, T_{I}$ and $T_{D}$. The smaller ISTE is get, the better PI-PD controller is designed. Thus there are four controller gains to be optimized. Those four parameters are tuned by mean of GA as explained in the next section.

\section{AQM USING PI-PD CONTROLLER}

Geneticalgorithm is an artificial optimization technique developed in analogy to natural evolution performing an exploration of the search space. It is considered as an efficient scheme for searching the global or near global solution of complex optimization problems.

The common GA problem is composed of three operations: reproduction, crossover and mutation. In the beginning an initial chromosome is randomly generated. These chromosomes are candidate solution of the problem. 
Then in the reproduction phase the fitness value of each chromosome is assessed. Further, this value is used in the selection process to provide bias toward fitter individual. Once the selection process is complete, the crossover algorithm is initiated. The crossover operation swaps certain parts of the two selected strings in a bid to capture the good parts of old chromosomes and create a better new one. Mutation is the occasional random alternation of a value of a string position. The probability of mutation is normally low because a high mutation rate would destroy fit strings and degenerate the genetic algorithm to random search.

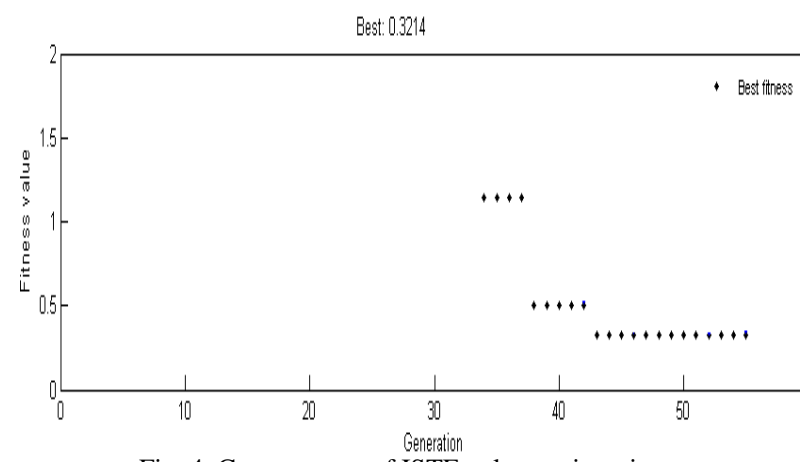

Fig. 4. Convergence of ISTE value vs. iteration.

For a TCP/AQM network modelled by Eq. (1), it is assumed that $N=60$ homogeneous TCP connections and shares one bottleneck link with a capacity of $15 \mathrm{Mbps}$. Furthermore, the propagation delay of the bottle link capacity and round trip time (RTT) were $0.2 \mathrm{~s}$ and $0.246 \mathrm{~s}$ respectively, the desired queue size was $q_{0}=200$ packets. The chromosome comprising four genes represent a set of parameters $K_{P}, K_{f}, T_{I}$ and $T_{D}$. In this simulation PI-PD controller gains are optimized with a fixed population size of 50 for 60 generation as stopping criterion. After a series of GA experiments, the best convergence curve of the ISTE value vs. iteration is shown in Fig. 4. The different parameters

$K_{P}=1.16 \times 10^{-7}, K_{f}=3.29 \times 10^{-3}, T_{I}=5.1 \times 10^{-5}$,

$T_{D}=0.26$

\section{Simulation Results}

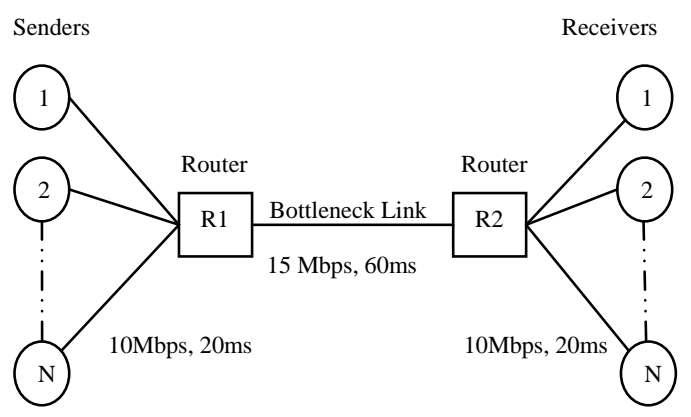

Fig. 5. Dumbell network topology.

Theperformance and effectiveness of the proposed GA PI-PD are verified in a series of numerical simulations using $n s-2$ (Network Simulator-2) [8] with sampling frequency of
$160 \mathrm{~Hz}$. under different circumstances. Queue buffer size of router is 800 packets. Our dumbbell network topology with each link capacity and propagation delay is depicted in Fig. 5.

\section{A. Experiment 1}

In our first experiment with the PI-PD controller we use a simple situation of only constant File Transmission Protocol (FTP) as senders and receivers. There are 60 FTP flows through bottleneck link to receiver. The queue length plots of both controllers are shown in Fig. 6. It is clear that PI-PD controller shows faster response and low overshoot in comparison to PI controller.

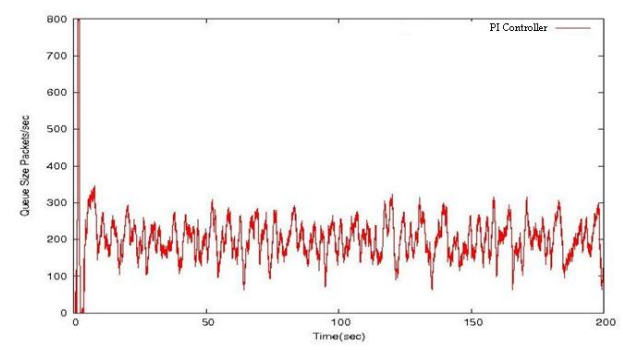

(a)

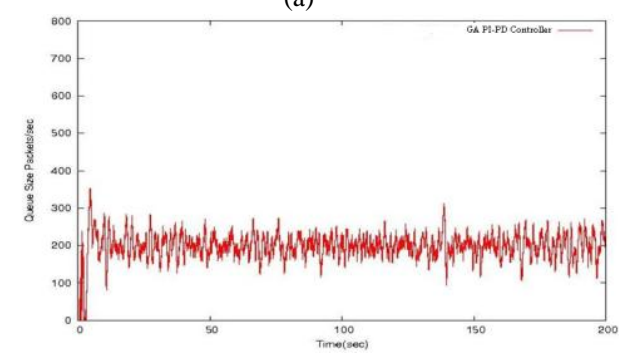

(b)

Fig.6. Comparison of PI and GA PI-PD controller under constant TCP connections.

\section{B. Experiment 2}

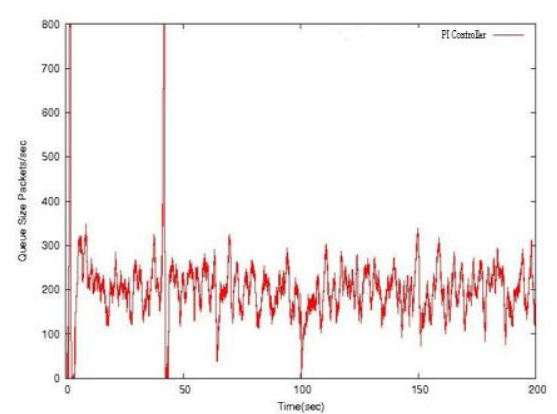

(a)

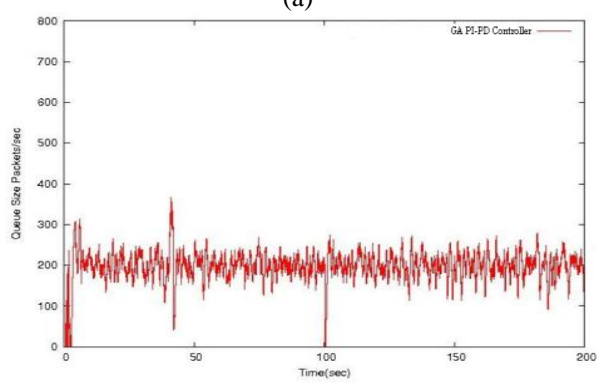

(b)

Fig. 7. Response of queue length under dynamic traffic change.

Now the disturbance of 20 TCP connections is introduced from 40 s to 100 s. It can be clearly observed from Fig. 7 that PI controller is not robust with respect to the variation in the 
load. Whereas, the proposed PI-PD controller efficiently manages the desired queue length and avoid queue-overflowing.

\section{Experiment 3}

Next, to consider more realistic scenarios, bursting and unresponsive flows were introduced into our simulations. In the dumbbell network topology we set the number of TCP flows to 60 and number of Constant Bit Rate (CBR) flows to 5. Each FTP flow was based on TCP; the CBR flow (bursting ON/OFF flow) is based on the User Datagram Protocol (UDP). The additional bursting CBR flows are active from 40 s to 100 s. The response of queue length using GA PI-PD and PI are depicted in Fig. 8. The result shows that GA PI-PD schemes can react rapidly and regulate the system effectively to achieve the desired queue length.

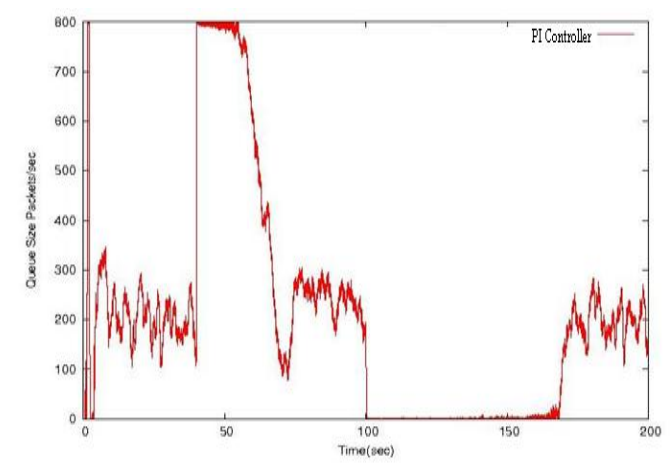

(a)

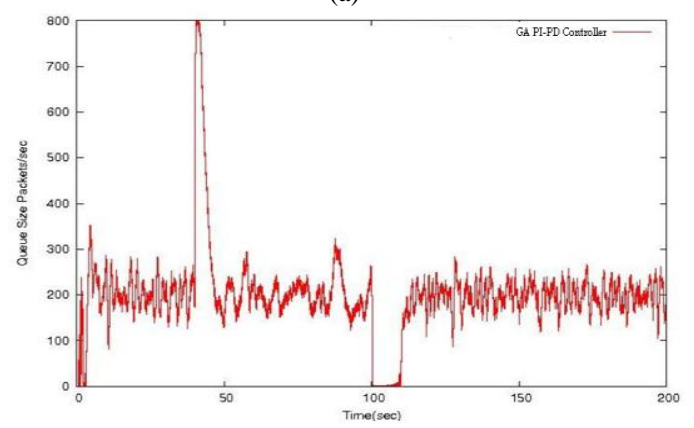

(b)

Fig. 8. Response of GA PI-PD and PI AQM schemes with bursting and unresponsive flows (FTP + UDP).

\section{CONCLUSION}

AGA-based PI-PD controller for AQM has been presented to ensure both high utilization and low packet loss rate by regulating the queue length in an internet router. Based on GA algorithm a simple and effective PI-PD controller has been proposed. Four PI-PD controller gains can be directly obtained by solving the specified optimization problem via genetic algorithm. The performance of proposed AQM

Congestion control scheme has been evaluated under different traffic scenarios via the $n s$ simulations. The simulation results reveal that the proposed scheme is superior to existing PI scheme, especially when there are UDP disturbances.

\section{FUTURE WORK}

However the new AQM schemes show better result than PI in simulation but its performance under real internet traffic scenarios is yet to be verified. Thus we are planning to set up a test bed in our institute to check different AQM scheme practically. Till now we have configured the Linux machine as a router in which the existing RED queue has been modified with new one. Real time simulation will be done using the TCP/UDP traffic generator software. Further, Smith Predictor can be used to get accurate tuning parameters of RED queue.

\section{ACKNOWLEDGMENT}

This research is supported by Department of Science and Technology (DST) New Delhi, India (Ref-SR/FTP/ETA-39/2007). The authors are thankful to Manish Shrivastava, Saket Saurav, and Rohitash Kumar of the Computer Science Department at PDPM-Indian Institute of Information Technology, Design \& Manufacturing, Jabalpur for their useful comments and fruitful discussions. Authors tried his best to cite maximum research papers used in this research project.

\section{REFERENCES}

[1] S. Floyd and V. Jacobson, "Random Early Detection gateway for congestion avoidance," IEEE/ACM Trans. on Networking, vol. 1, pp. 397-413, Aug. 1993.

[2] V. Misra, W. B. Gong, and D. Towsley, "Fluid-based analysis of a network of AQM routers supporting TCP flows with an application to RED," in Proc. ACM/SIGCOMM, 2000.

[3] C. V. Hollot, V. Misra, D. Towsley, and W. B. Gong, "On designing improved controllers for AQM routers supporting TCP flows," in Proc. IEEE INFOCOM, Alaska, 2001, pp. 1726-1734.

[4] Y. Li, K. T. Ko, and G. Chen, "A Smith predictor-based PI-controller for active queue management," IEICE Trans. on Communication, vol. 88, pp. 4293-4300, 2005.

[5] S. H. Low, F. Paganine, and J. C. Doyle, "Internet congestion control," IEEE Contr. Sys. Mag., vol. 22, pp. 28-43, Jan. 2002.

[6] E. C. Park, H. Lim, K. J. Park, and C. H. Choi, "Analysis and design of the virtual rate control algorithm for stabilizing queues in TCP networks," Computer Networks, vol. 44, pp. 17-41, 2004

[7] D. E. Goldberg, Genetic Algorithm in Search, Optimization, and Machine Learning, Reading, MA, Addison Wesley.

[8] Ns-2 Network Simulator. [Online]. Avaliable: http://www.isi.edu/nsnam/ns/

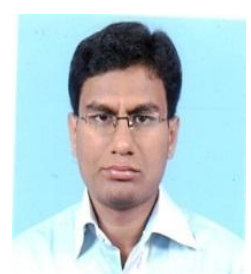

R. K. Sundaram was born in Patna, Bihar, India He received the bachelor of technology (B.Tech) degree in electronics and communication engineering from the PDPM IIITDM, Jabalpur, Madhya Pradesh, India. He is currently pursuing master of technology (M.Tech) degree from IIT Kanpur in the field of RF, microwav and photonics. His research interests include congestion control in satellite communication components.

TCP routers and multipaction in high power

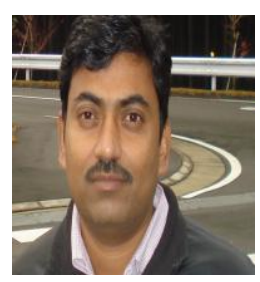

P. K. Padhy was born in Orissa, India. He received the $\mathrm{Ph} . \mathrm{D}$. degree from IIT Guwahati in 2007. He is currently an associate professor in the discipline of Electronics and Communication Engineering at PDPM IIITDM, Jabalpur, Madhya Pradesh, India. His research interests include automatic controller tuning, identification and control of processes and robotics. 